\title{
Obtención de semilla híbrida de melón (Cucumis melo L.) en invernadero
}

\section{Obtaining hybrids seed of melo (Cucumis melo L.) in greenhouse}

\author{
Gabriel-Ortega Julio $^{1 *(D)}$, Burgos-López Gema ${ }^{2}(\mathbb{D})$ Barahona-Cajape Nora $^{3}(\mathbb{D})$ Castro-Piguave Carlos $^{1}(\mathbb{D})$, \\ Vera-Tumbaco Máximo ${ }^{1}$, Morán-Morán Jéssica ${ }^{1}$ (D)
}

\section{Datos del Artículo \\ ${ }^{1}$ Facultad de Ciencias Naturales y de la Agricultu- ra. \\ Universidad Estatal del Sur de Manabí (UNESUM). \\ km 1.5 vía Noboa, Campus los Ángeles, Jipijapa. Manabí, Ecuador. \\ ${ }^{2}$ Consultora de Portoviejo, Manabí, Ecuador. \\ ${ }^{3}$ Consultora de Manta, Manabí, Ecuador. \\ *Dirección de contacto \\ Facultad de Ciencias Naturales y de la Agricultura. \\ Universidad Estatal del Sur de Manabí \\ (UNESUM). km 11/2 vía Noboa s/n. \\ Campus los Ángeles, Jipijapa, Ecuador \\ Telf: +05-2600229. \\ Julio Gabriel-Ortega \\ E-mail address : Julio.gabriel@unesum@edu.ec i.gabriel@proinpa.org}

\section{Palabras clave:}

Producción

tolerancia,

resistencia,

volumen,

peso,

peso,

calidad, frutos

J. Selva Andina Res. Soc. 2021; 12(1):38-51.

ID del artículo: 147/JSARS/2020

\section{Historial del artículo.}

Recibido septiembre 2020 Devuelto noviembre 2020.

Aceptado noviembre 2020

Disponible en línea, febrero 2021.

\begin{tabular}{c} 
Editado por: \\
Selva Andina \\
Research Society \\
\hline
\end{tabular}

\section{Resumen}

La presente investigación fue realizada en Puerto La Boca, provincia Manabí, Ecuador, en el año 2019. El objetivo fue obtener semilla híbrida $F_{1}$ de melón bajo condiciones de invernadero. Para el desarrollo de esta investigación se implementó en un invernadero de $500 \mathrm{~m}^{2}$ una parcela en un diseño experimental de bloque completos aleatorizados con tres repeticiones y dos tratamientos $\left(\mathrm{A}_{1}\right.$ : plantas con poda y $\mathrm{A}_{2}$ : plantas sin poda). Las variables de respuesta fueron: altura de plantas $(\mathrm{m})$, diámetro de tallo $(\mathrm{mm})$, número de nudos antes del fruto, número de nudos después del fruto, volumen del fruto $\left(\mathrm{cm}^{3}\right)$, peso de frutos $(\mathrm{g})$, número de semillas vivas, número de semillas muertas, peso de semillas con embrión ( $\mathrm{g}$ ), y una estimación del costo/beneficio mediante presupuestos parciales. Los resultados mostraron que el mejor promedio de peso del fruto, número de semillas vivas y peso de semilla viable fue para el tratamiento con poda. El análisis de presupuestos parciales mostró que la producción de semilla fue altamente rentable para los tratamientos evaluados con una relación beneficio costo $\mathrm{B} / \mathrm{C}>1$, siendo el tratamiento con poda la mejor. La poda mejoró la producción de semilla híbrida $F_{1}$ de melón, asimismo mejoró la calidad de los frutos.

2021. Journal of the Selva Andina Research Society ${ }^{\circledR}$. Bolivia. Todos los derechos reservados.

\section{Abstract}

This research was carried out in Puerto La Boca, Manabí province, Ecuador, in 2019. The objective was to obtain hybrid F1 melon seed under greenhouse conditions. For the development of this research, a plot was implemented in a $500 \mathrm{~m} 2$ greenhouse in a randomized complete block experimental design with three repetitions and two treatments (A1: Plants with pruning and A2: Plants without pruning). The response variables were: plant height $(\mathrm{m})$, stem diameter $(\mathrm{mm})$, number of nodes before fruit, number of nodes after the fruit, fruit volume (cm3), fruit weight (g), number of live seeds, number of dead seeds, the weight of seeds with the embryo (g), and a cost/benefit estimate using partial budgets. The results showed that the best average fruit weight, number of live seeds, and viable seed weight was for the pruning treatment. The partial budget analysis showed that seed production was highly profitable for the treatments evaluated with a cost-benefit ratio $\mathrm{B} / \mathrm{C}>1$, with the pruning treatment being the best. Pruning improved melon F1 hybrid seed production, as well as fruit quality.

\section{Keywords:}

\section{Production,}

tolerance,

resistance,

volume,

weight,

lines,

$\mathrm{F}_{1}$,

quality,

fruits.

2021. Journal of the Selva Andina Research Society ${ }^{\circledR}$. Bolivia. All rights reserved. 


\section{Introducción}

El origen del melón es ampliamente disputado, hay argumentos que indican que se originó en el sur de Asia o África ${ }^{1-3}$. El sur de Asia tiene una gran diversidad de cultivares de melón, las especies de $\mathrm{Cucu}$ mis tienen un número cromosómico $\mathrm{n}=12$, excepto Cucumis hystrix Chakrav, se origina en África y se les conoce como "el grupo africano" ${ }^{4}$. De las 32 especies de Cucumis, 31 tienen un número cromosómico $n=12^{\frac{1,5}{5}}$. Aunque los últimos estudios moleculares sistemáticos sugieren Asia como su origen ${ }^{6}$.

Actualmente, China y Estados Unidos tienen la mayor producción de melón en el mundo ${ }^{7}$, desde 2014-2017 se estima que se produjo 29626 millones de kg en 1.19 millones de ha. China produjo 14752 millones de kg, Turquía 1707 millones de kg e Irán 1476 millones de $\mathrm{kg}^{7}$. El dulzor de la fruta, sabor o aroma, textura y fitonutrientes son los rasgos más importantes que los consumidores prefieren ${ }^{8}$.

El Ecuador dispone de condiciones ambientales favorables para el cultivo de una infinidad de especies hortícolas, tanto en la Sierra como en la Costa. En el Ecuador se dedican unas 40000 ha de hortalizas, la cebolla colorada ocupa 7920 ha, tomate riñón 7560 ha, cebolla blanca 4230 ha, sandía 3860 ha, melón 3430 ha y zanahoria amarilla 2800 ha ${ }^{2}$. En el país la explotación de melón (Cucumis melo L.) en las últimas décadas tuvo un auge notable, ocupando el segundo lugar por superficie sembrada entre las cucurbitáceas ${ }^{10}$. En el litoral ecuatoriano se cultiva desde diciembre hasta marzo (época invernal), una superficie de 924 ha con una producción de 7549 t, se exporta a los países europeos el $1.4 \%$ del total de la producción.

En Manabí el cultivo del melón está ampliamente difundido, y los cultivares híbridos más comúnmente utilizados son: Edisto 47, Pacstar, Primo y Excelsior $^{11}$. El recinto Cantagallo ubicado a $4 \mathrm{~km}$ del filo costero del Cantón Jipijapa, Provincia de Manabí, cuenta con un microclima especial, apto para el desarrollo de cualquier tipo de cultivo de ciclo corto y perenne, la mayoría de sus habitantes cuentan con terrenos propios para sembrar existe abundantes fuentes de agua subterránea, en estos sitios se cultiva el melón, sandía, cebolla, etc $\underline{11}$.

Por otra parte, se sabe que, desde la antigüedad el melón fue sometido al proceso de mejoramiento genético, mediante el uso de métodos tradicionales de reproducción ${ }^{12}$. Debido a las fuertes barreras de incompatibilidad sexual interespecífica e intergenérica, el potencial genético quedó restringido el desarrollo de nuevos cultivares mejorados de melón ${ }^{\underline{13}}$. Así el mejoramiento genético de melón por hibridación es relativamente lenta y restringido a un grupo de pocos genes $\frac{14}{}$. Sin embargo, el desarrollo de plantas mediante el uso de herramientas biotecnológicas ofrece la posibilidad de obtener nuevos cultivares, más rápidamente, evitando las barreras genéticas naturales, asimismo se desarrollaron métodos biotecnológicos, para aumentar la diversidad genética utilizando herramientas de hibridación somática o de transferencia de genes mediante la construcción de mapas genéticos $\underline{15}, \underline{16}$.

La relación genética y el fenotipo son factores importantes que gobiernan la expresión de la heterosis en la progenie híbrida de muchas especies de plantas de polinización cruzada, y no es comprendida plenamente en el melón ${ }^{17}$. La Aptitud Combinatoria General (ACG), específica (ACE) y el análisis de heterosis se pueden utilizar para investigar la respuesta heterótica ${ }^{18,19}$. Así también se utilizaron marcadores moleculares para estudiar la diversidad genética y su relación con la heterosis en diferentes especies de plantas. Xiao et al ${ }^{20}$ demostró la existencia de una estrecha relación entre la diversidad 
genética y el rendimiento de híbridos y heterosis en arroz. Existe un acuerdo general en que la ACG y los efectos heteróticos asociados son factores fundamentales en la selección de los padres del melón híbrido $^{21,22}$. Además, la heterosis, en función del rendimiento, es a menudo relacionado con el grado de parentesco genético y está fuertemente influenciado por las condiciones del cultivo $\mathrm{o}^{\underline{21}, \underline{22}}$.

Se debe mencionar que los agricultores de Puerto La Boca están organizados en una asociación denominada "Asociación Agroartesanal Puerto La Boca Cantagallo", y cuentan con 54 invernaderos que cubren 2.75 ha de superficie, que los convierte en un sitio potencial y apreciable, para la producción de semillas $\underline{23}$.

El objetivo de la investigación fue obtener semilla híbrida $F_{1}$ de melón bajo condiciones de invernadero.

\section{Materiales y métodos}

Ubicación. La investigación fue desarrollada entre junio a octubre 2019, en un invernadero de $500 \mathrm{~m}^{2}$ ubicado en el Recinto Puerto La Boca de la Parroquia Puerto Cayo, Cantón Jipijapa, que está ubicado a 45 min de Jipijapa $\left(1^{\circ} 18^{\prime} 20^{\prime \prime} \mathrm{S}, 80^{\circ} 45^{\prime} 42^{\prime \prime} \mathrm{O}\right.$ y altura de $53 \mathrm{msnm}$ ), su clima posee una temperatura de $24.8{ }^{\circ} \mathrm{C}$, la precipitación promedio anual es de 298 $\mathrm{mm}$, concentrándose la mayor cantidad de lluvia en el mes de febrero, mientras que el mes más seco es $\operatorname{agosto}^{24}$.

Tratamientos y diseño experimental. En la línea parental hembra H20583, se aplicó dos tratamientos $\left(\mathrm{A}_{1}\right.$ : plantas con poda y $\mathrm{A}_{2}$ : plantas sin poda). Se eligieron en cada unidad experimental (UE) 20 plantas al azar, las cuales fueron marcadas para su evaluación.

La parcela de investigación fue implementada en un diseño experimental de bloques completamente aleatorio (DBCA), con tres repeticiones, y dos tratamientos ${ }^{25}$. En el experimento se bloqueó el efecto de la época de polinización (25, 30 y 35 días), debido a que no fue posible realizar todas las polinizaciones en una misma fecha.

Análisis estadísticos. Sobre la base del modelos definido y previo análisis de normalidad y homogeneidad de varianza, se realizó análisis de varianza (ANVA)para datos desbalanceados, para probar hipótesis de los efectos fijos, así como las comparaciones de medias de los tratamientos mediante la prueba de tukey al $\mathrm{Pr}<0.05$ de probabilidad. El ANVA de datos desbalanceados también sirvió para estimar los componentes de varianza para los efectos aleatorios. Los análisis indicados se realizaron utilizando el Proc GLM del SAS ${ }^{26}$.

Análisis de correlación. Entre las variables de respuesta correspondiente utilizando el coeficiente de correlación de Pearson ${ }^{25}$.

Variables de respuesta. i) Altura de plantas (m) (AP). Se midió la altura utilizando una cinta métrica, considerando desde la base de cuello al nivel del suelo hasta el ápice de la planta. ii) Diámetro de tallo $(\mathrm{mm})$ (DT). Se utilizó un calibrador vernier, midiendo a $15 \mathrm{~cm}$ de altura de suelo el tallo principal. iii) Número de nudos antes del fruto (NDNAF). Se contabilizó el número de nudos antes del fruto. iv) Número de nudos después del fruto (NDNDF). Se contabilizó el número de nudos después del fruto. v) Largo, ancho y alto del fruto $(\mathrm{cm})$. Los datos de los frutos se midieron con la ayuda de un calibrador vernier considerando cada uno de los frutos de la parcela útil. vi) Peso de frutos (g) (PF). Los frutos cosechados dentro de la parcela útil se pesaron con una balanza digital calibradora en g. vii) Número de semillas. Una vez recolectado el fruto en estado maduro (fácil desprendimiento de la planta) se cortó de forma longitudinal para extraer las semillas con una cuchara sopera y se almacenaron en 
una funda transparente para su fermentación, al cabo de $30 \mathrm{~h}$ se lavaron con mucho cuidado con agua potable, se colocaron todas las semillas limpias en un balde para posteriormente seleccionar las semillas viables, se consideraron a aquellas que se quedan en la base del balde semillas viables, mientras las que flotaban se consideró semillas carentes de embrión. Luego de la selección se procedió a secar las semillas a temperatura ambiente para posteriormente almacenarlas en sobre de papel. viii) Peso de semillas con embrión ( $g$ ). Las semillas obtenidas en cada fruto se pesaron en una balanza gramera digital teniendo cuidado para no mezclar. ix) Volumen total de frutos $\left(\mathrm{cm}^{3}\right)$. Para determinar el volumen de cada fruto se utilizó la formula recomendada por Moreno ${ }^{27}$.

Beneficio/Costo. Para la relación B/C se determinó el beneficio de la tecnología, en su interpretación se consideró si el resultado fue mayor a $1^{\frac{28}{2}}$ este fue aceptable o rentable, si el resultado fue igual a 1, lo que indicó fue que no tuvo beneficio de lucro ni pérdida y si el resultado fue menor a 1 implicó que no fue rentable, por lo cual el proyecto sería rechazado.

Manejo de la investigación. Los tratamientos $\mathrm{A}_{1} \mathrm{y}$ $\mathrm{A}_{2}$, fueron realizados en el parental femenino. Los tratamientos fueron distribuidos en un DBCA con 20 UE. El diseño permitió bloquear el efecto del tiempo de polinización $(25,30,35$ días después del trasplante). Cada hilera tuvo 110 plantas por tratamiento, de las cuales fueron seleccionadas 10 plantas al azar en cada repetición, para evaluar las variables de respuesta. En total se evaluaron 60 plantas. La distancia de trasplante fue de $0.30 \mathrm{~m}$ entre plantas y $1.5 \mathrm{~m}$ entre hileras. El experimento tuvo 1320 plantas.

La preparación del suelo fue manualmente. Se realizó la remoción y el desterronado de las platabandas. Se aplicó materia orgánica (biocompost) a razón de
$75 \mathrm{~kg}$ por hilera de $33 \mathrm{~m}$ de largo. Luego se formó las platabandas de $0.50 \mathrm{~m}$ de ancho por $33 \mathrm{~m} \mathrm{de}$ largo.

Para la germinación de la semilla, se preparó el sustrato con biocompost, hoja de guaba y tierra del lugar, en una proporción 2:1:1. Se puso $10 \mathrm{~kg}$ de humus y una bolsa ( $10 \mathrm{~g})$ de micorriza para evitar el ataque de patógenos que causan mal de almácigo (damping off).

Una vez preparado el sustrato se procedió a llenar los hoyos con este, teniendo cuidado de humedecerla óptimamente. Luego fueron sembradas las semillas de los parentales en estas bandejas.

El riego de las bandejas se realizó dos veces por día para mantener la humedad. Para prevenir ataque de enfermedades se aplicó un fungicida de amplio espectro. El trasplante definitivo se realizó en hileras, para lo que se cavaron hoyos con una profundidad de $0.15 \mathrm{~m}$, después se procedió al trasplante de una planta por hoyo.

Para el control del mildiu velloso causada por el oomycete Pseudoperonospora cubensis se realizó la aplicación de Metalaxy+Mancozeb (Ridomil Gold 68 WP) a los 8 y 30 días después del trasplante en una dosis $70 \mathrm{~g} / 15 \mathrm{~L}$ de agua ${ }^{23}$.

Para el control de insectos-plaga como mosca blanca (Bemisia tabaci), negrita (Prodiplosis longifila) y pulgones (Myzus persicae), se utilizó Acetamiprid a los 10 días después del trasplante (ddt) con una dosis de $40 \mathrm{~g} / 15 \mathrm{~L}$ de agua. Asimismo, se aplicó Bacillus thuringiensis a los 20 y 50 ddt en una dosis de $80 \mathrm{~g} / 15 \mathrm{~L}$ de agua.

La poda se realizó en una sola rama principal y eliminando las ramas restantes. Se eliminaron las hojas viejas y los brotes para evitar la formación de otras ramas secundarias. El tutorajes se realizó después de la poda, así mismo después de cada poda se trató con un fungicida de contacto para evitar enfermeda- 
des por las heridas causadas por esta labor, el riego fue por goteo.

Manejo de los cruzamientos. El parental macho que fue sembrado dos semanas antes en dos surcos centrales, se colectó flores masculinas en tarrinas plásticas, dejando secar entre media hora a una hora. Luego se trasladó hasta las plantas madre. En las plantas madre se eligió las flores no abiertas, mismas que aún no fueron polinizadas. Estas flores fueron emasculadas y liberadas de su corola cuidadosamente con una pinza quirúrgica. Luego, se frotó con las anteras de la flor colectada, los estigmas de la flor hembra, dejándolas totalmente empapada y de color amarillo. Posteriormente se tapó la flor con sumo cuidado con una cinta para que no sea contaminada con polen foráneo, para así garantizar el cruzamiento. Se polinizó entre dos a cinco flores por planta. Cuando cuajo la flor y se evidenció la formación del fruto, se hizo un aclareo, eliminando los frutos más pequeños, dejando solamente uno o dos frutos por planta. Este proceso se repitió durante tres oportunidades, a los 25, 30 y 35 días. Este trabajo fue realizado principalmente por mujeres, que lograron un $100 \%$ de prendimiento.

Cosecha y poscosecha de melón. La cosecha se la realizó a partir de los 120 dds del cultivo de melón.
En esta etapa se recolectó los frutos según sus grados de madurez para obtener semillas híbridas, en el cual se recogió un fruto por planta.

Análisis económico. Para el análisis económico se consideró los costos que varían, según las recomendaciones de Boardman ${ }^{28}$. En este análisis no se consideró los costos fijos, sino solamente los costos variables y durante todo el ciclo del cultivo. Se consideraron los costos de semilla, trasplante, poda, plaguicidas, fertilizantes, aplicaciones de los tratamientos y fertilizantes, mano de obra utilizada en la cosecha, la venta del producto, etc.

\section{Resultados}

Se observó que en general todas las variables evaluadas manifestaron una curva asimétrica y platicúrtica, y que en general las variables de respuesta revelaron coeficientes de variación (C.V.), que están en el rango permitido para este tipo de investigaciones. La prueba de kosmogorov-Smirnov ${ }^{29}$, expuso que no hubo significancia al $\operatorname{Pr}<0.01$ de probabilidad. Por lo que se asumió que las variables de respuesta evaluadas exhibieron distribución normal (tabla 1), con coeficientes de variación recomendados para este tipo de investigación.

Tabla 1 Análisis de normalidad de las variables en estudio

\begin{tabular}{|c|c|c|c|c|c|c|c|c|c|}
\hline & $\mathbf{A P}$ & DT & NDNAF & NDNDF & VFr & PF & NSV & NSM & PS \\
\hline Desviación & 0.19 & 0.78 & 4.15 & 2.35 & 718471.14 & 143.36 & 177.4 & 14.04 & 2.50 \\
\hline Varianza & 0.03 & 0.61 & 17.30 & 5.53 & 5.16 & 20553.8 & 31488.5 & 197.3 & 6.29 \\
\hline Asimetría & 0.01 & 0.28 & -0.30 & 0.65 & -0.42 & -0.27 & 0.22 & 0.31 & 0.20 \\
\hline Curtosis & -0.81 & -0.14 & 0.71 & -0.24 & -0.90 & -1.39 & -1.63 & -0.29 & 0.22 \\
\hline C.V & 11.17 & 24.10 & 21.05 & 22.64 & 28.52 & 31.22 & 18.31 & 22.64 & 36.01 \\
\hline Kolmogórov-Smirnov P Valor & 0.04 & $<0.01$ & $>0.15$ & $<0.01$ & $>0.15$ & $>0.15$ & $<0.01$ & $>0.15$ & 0.12 \\
\hline
\end{tabular}

**: Altamente significativo al $\operatorname{Pr}<0.01$ de probabilidad, *: Significativo al $\operatorname{Pr}<0.05$ de probabilidad, ns: no significativo, altura planta (AP), diámetro del tallo (DT), número de nudos antes del fruto (NDNAF), número de nudos después de fruto (NDNDF), volumen del fruto (VFr), peso del fruto (PF), número de semillas vivas (NSV), número de semillas muertas (NSM) y peso de semillas (PS).

Análisis de homogeneidad de varianzas. El análisis de homogeneidad de varianzas indica que las medias fueron homogéneas (tabla 2), la prueba de Chi cuadrada (ChiSq) no fue estadísticamente significativo al $\operatorname{Pr}<0.01$ de probabilidad, lo que corroboró la continuidad de los análisis de varianza. 
Tabla 2 Análisis de homogeneidad de varianza

\begin{tabular}{lll}
\hline Chi-cuadrado & DF & Pr $>$ ChiSq \\
\hline 0.000000 & 253 & 1.00 \\
\hline
\end{tabular}

Análisis de varianza. El análisis de varianza de las variables AP, DT y NDNAF, reveló que los coeficientes de variación (8.76 a 20.21) están dentro de los rangos permitidos para este tipo de investigaciones. La variable AP expuso diferencias altamente significativas al $\operatorname{Pr}<0.01$ de probabilidad, lo que estaría indicando que al menos uno de los tratamientos fue diferente (tabla 3 ).

Tabla 3 Análisis de varianza de las variables estudiadas

\begin{tabular}{ccccc}
\hline \multirow{2}{*}{ Fv } & \multirow{2}{*}{ Gl } & \multicolumn{3}{c}{ Cuadrados medios } \\
\cline { 3 - 5 } & & AP & DT & NDNAF \\
\hline Total & 52 & & & \\
Repetición & 2 & $0.01 \mathrm{~ns}$ & $4.12^{* *}$ & $55.46^{*}$ \\
Tratamiento & 1 & $0.76^{* *}$ & $0.88 \mathrm{~ns}$ & $1.32 \mathrm{~ns}$ \\
Error & 49 & 0.02 & 0.47 & 15.95 \\
C.V.(\%) & & 8.76 & 21.21 & 20.21 \\
\hline
\end{tabular}

**: Altamente significativo al $\operatorname{Pr}<0.01$ de probabilidad, *: Significativo al $\operatorname{Pr}<0.05$ de probabilidad, ns: no significativo. Altura de planta (AP), diámetro de tallo (DT) y número de nudos antes del fruto (NDNAF)
El análisis de la variable NDNDF no fue significativo al $\operatorname{Pr}<0.05$ de probabilidad (tabla 4).

Tabla 4 Análisis de varianza para el número de nudos después del fruto

\begin{tabular}{cccccc}
\hline Origen & gl & SC & CM & Valor F & Pr>F \\
\hline Total & 52 & & & & \\
Repetición & 2 & 2.20 & $1.10^{* *}$ & 3.65 & 0.03 \\
Tratamiento & 1 & 0.18 & 0.18 & $0.62 \mathrm{~ns}$ & 0.43 \\
Error & 49 & & 0.30 & &
\end{tabular}

C.V. (\%) $\quad 22.64$

**: Altamente significativo al $\operatorname{Pr}<0.01$ de probabilidad, *: Significativo al $\operatorname{Pr}<0.05$ de probabilidad, ns: no significativo.

Tabla 5 Análisis de varianza para el volumen del fruto

\begin{tabular}{cccccc}
\hline Origen & gl & SC & CM & $\begin{array}{c}\text { Valor } \\
\text { F }\end{array}$ & Pr $>\mathbf{F}$ \\
\hline Total & 27 & & & & \\
Repetición & 2 & 2.00 & 1.00 & 2.34 & 0.1183 \\
Tratamiento & 1 & 0.26 & 2.11 & $4.93 *$ & 0.0361 \\
Error & 24 & 2.11 & 429412514366 & & \\
C.V.(\%) & 26.01 & & & & \\
\hline **: Altamente significativo al $\operatorname{Pr}<0.01$ de probabilidad, *: Significativo al $\operatorname{Pr}<0.05$
\end{tabular}
de probabilidad, ns: no significativo

Tabla 6 Análisis de varianza para el peso del fruto

\begin{tabular}{cccccc}
\hline Origen & gl & SC & CM & Valor F & Pr>F \\
\hline Total & 22 & & & & \\
Repetición & 2 & 218224.90 & 109112.45 & 10.16 & 0.0010 \\
Tratamiento & 1 & 25702.70 & 25702.70 & $2.39 \mathrm{~ns}$ & 0.13 \\
Error & 19 & & 10734.46 & & \\
C.V.(\%) & 22,56 & & & & \\
\hline
\end{tabular}

**: Altamente significativo al $\operatorname{Pr}<0,01$ de probabilidad, *: Significativo al $\operatorname{Pr}<0,05$ de probabilidad, ns: no significativo.

El análisis de varianza para la variable VFr expuso diferencias significativas al $\mathrm{P}<0.05$ de probabilidad (tabla 5).

El análisis de varianza para la variable PF no expuso diferencia significativa al $\operatorname{Pr}<0.05$ de probabilidad para tratamientos (tabla 6).

El análisis de varianza para la variable NSV señala diferencias significativas al $\operatorname{Pr}<0.05$ de probabilidad para tratamientos, mientras el NSM no señala significancia (tabla 7).

Tabla 7 Análisis de varianza para las variables en estudio

\begin{tabular}{cccc}
\hline \multirow{2}{*}{ Fv } & gl & \multicolumn{2}{c}{ Cuadrados medios } \\
\cline { 3 - 4 } & & NSV & NSM \\
\hline Total & 37 & & \\
Repetición & 2 & $12.70 \mathrm{~ns}$ & $1.10^{*}$ \\
Tratamiento & 1 & $59.55^{*}$ & $0.18 \mathrm{~ns}$ \\
\hline
\end{tabular}




\begin{tabular}{|c|c|c|c|c|c|c|c|c|c|}
\hline & $\begin{array}{c}\text { Error } \\
\text { C.V. }(\%)\end{array}$ & 34 & $\begin{array}{r}9.9 . \\
18.3 \\
\end{array}$ & $\begin{array}{r}0.30 \\
22.6 \\
\end{array}$ & & & El a & $\begin{array}{l}\text { isis de } \\
\text { iferenc }\end{array}$ & $\begin{array}{l}\text { rianza p } \\
\text { sionifica }\end{array}$ \\
\hline & $\begin{array}{l}\text { **: Altament } \\
\text { *: Significati } \\
\text { Número de }\end{array}$ & $\begin{array}{l}\text { gnific } \\
1 \mathrm{Pr}< \\
\text { illas }\end{array}$ & $\begin{array}{l}\text { o al Pr } \\
\text { de pr } \\
\text { as }(\mathrm{N}\end{array}$ & $\begin{array}{l}.01 \text { de probabili } \\
\text { abilidad, ns: no } \\
\text { I) y número de }\end{array}$ & $\begin{array}{l}\text { ficativo. } \\
\text { nillas } \mathrm{m}\end{array}$ & & lidac & ara trat & hientos \\
\hline & & & & Tabla 8 A & lisis d & arian & para & eso de & nillas \\
\hline & & & & Origen & gl & SC & CM & Valor F & $\operatorname{Pr}>\mathbf{F}$ \\
\hline & & & & Total & 30 & & & & \\
\hline & & & & Repetición & 2 & 8.987 & 4.493 & 0.79 & 0.4644 \\
\hline & & & & Tratamiento & 1 & 22.26 & 22.261 & $3.91 \mathrm{~ns}$ & 0.0583 \\
\hline & & & & Error & 27 & & 5.694 & & \\
\hline & & & & C.V. (\%) & 34.25 & & & & \\
\hline
\end{tabular}

Análisis de medias. El análisis de medias, mediante la comparación múltiple de tukey para la variable AP manifestó diferencias significativas al $\operatorname{Pr}<0.05$ de probabilidad, indicando que $\mathrm{A}_{2}$, fue el que más altura expuso, lo cual es lógico, desde que se hizo un despunte de los ápices de las plantas con poda (tabla 9).

Para el DT, NDNAF, NDNDF, VFr, PF y MSM, no expusieron diferencias significativas al $\operatorname{Pr}<0.05$ de probabilidad entre los tratamientos en la comparación de sus medias. Indicando esto, que todas fueron estadísticamente iguales.

\section{Tabla 9 Prueba del rango múltiple de Tukey para las} variables en estudio

\begin{tabular}{|c|c|c|c|}
\hline Tratamiento & $\mathbf{A P}$ & NSV & PS \\
\hline Con poda & $1.59 \mathrm{~b}$ & $303.14 \mathrm{a}$ & $7650 a$ \\
\hline Sin poda & $1.83 \mathrm{a}$ & $85.38 \mathrm{~b}$ & $5727 \mathrm{~b}$ \\
\hline DSH & 0.08 & 95.21 & 1.84 \\
\hline
\end{tabular}

Para el NSV, se observó diferencias significativas al $\operatorname{Pr}<0.05$ de probabilidad (tabla 9), entre los tratamientos, siendo $\mathrm{A}_{1}$ el mejor, exponiendo un promedio de 303 semillas/fruto, respecto de 85 semillas/fruto.
Para el PS, se observó diferencias significativas al $\operatorname{Pr}<0.05$ de probabilidad (tabla 9), entre los tratamientos, siendo $\mathrm{A}_{1}$ el mejor, que manifestó un promedio de $7650 \mathrm{~g}$ de semillas, respecto de $5727 \mathrm{~g}$ de semillas del $\mathrm{A}_{2}$.

Análisis de correlación. En el análisis de Pearson (tabla 10), se observaron correlaciones altas y significativas para las variables DT versus PF (0.77) esto indicaría que a mayor DT mayor PF posteriormente VFr versus el PF (0.85), NSV (0.69) respecto del PS (0.51) esto evidenciaría que a mayor VFr mayor PF los mismo ocurrió con el NSV y PS. En el PF versus NSV (0.79) y PS (0.68), se estimó que a mayor PF hubo mayor NSV y PS. Visualizando el NSV con PS (0.58), estaría mostrando que a NSV mayor PS (tabla 10).

Análisis del Beneficio/Costo de los tratamientos. El análisis de rentabilidad de las estrategias para producción de semillas $F_{1}$ (tabla 11), manifestó que las alternativas que se utilizaron fueron rentables con una relación beneficio/costo mayor a $1(\mathrm{~B} / \mathrm{C}>1)$, Se distinguieron los tratamientos con poda con USD 12.998 y sin poda con USD 2.927 (tabla 11). 


\section{Discusión}

Para un fitomejorador el conocimiento adecuado del sistema de producción de las plantas con que trabaja es esencial, ya que esto define en gran medida el diseño genético y de apareamiento que se debe utilizar, así como el sistema de selección que debe emplearse $^{\underline{30}}$. En el caso de C. melo a pesar de ser una planta alógama desde el punto de vista del mejoramiento genético se maneja como una planta autógama, impidiendo el cruzamiento libre de esta especie a través del sellado de las flores, ya que de esta forma se produce un mayor avance en los programas

de mejoramiento ${ }^{\underline{30}}$.

Tabla 10 Análisis de correlación mediante el coeficiente de Pearson

\begin{tabular}{|c|c|c|c|c|c|c|c|c|c|}
\hline & AP & DT & NDNAF & NDNDF & VFr & PF & NSV & NSM & PS \\
\hline $\mathbf{A P}$ & $\begin{array}{c}1.0 \\
0\end{array}$ & 0.14 & $0.47 * *$ & -0.09 & -0.01 & 0.01 & -0.31 & -0.00 & 0.04 \\
\hline DT & & 1.00 & -0.08 & 0.08 & 0.46 & $0.77 * *$ & 0.20 & 0.19 & 0.28 \\
\hline NDNAF & & & 1.00 & $-0.68 * *$ & -0.04 & 0.00 & 0.02 & 0.08 & 0.28 \\
\hline NDNDF & & & & 1.00 & 0.06 & 0.16 & 0.11 & -0.18 & 0.06 \\
\hline VFr & & & & & 1.00 & $0.85 * *$ & $0.69 * *$ & 0.23 & $0.51 * *$ \\
\hline PF & & & & & & 1.00 & $0.79 * *$ & $0.53 *$ & $0.68 * *$ \\
\hline NSV & & & & & & & 1.00 & 0.14 & $0.58 * *$ \\
\hline NSM & & & & & & & & 1.00 & 0.00 \\
\hline PS & & & & & & & & & 1.00 \\
\hline
\end{tabular}

**: Altamente significativo al $\operatorname{Pr}<0.01$ de probabilidad, *: Significativo al $\operatorname{Pr}<0.05$ de probabilidad, ns: no significativo, altura planta (AP), diámetro del tallo (DT), número de nudos antes del fruto (NDNAF), número de nudos después de fruto (NDNDF), volumen del fruto (VFr), peso del fruto (PF), número de semillas vivas (NSV), número de semillas muertas (NSM) y peso de semillas (PS).

Tabla 11 Rentabilidad de los tratamientos para obtención de semillas $\mathbf{F}_{1}$

\begin{tabular}{|c|c|c|c|c|c|c|c|c|c|}
\hline Tratamiento & $\mathrm{NP}(1000 \mathrm{~m} 2)$ & $\begin{array}{c}\text { Número de } \\
\text { semillas/fruto }\end{array}$ & $\begin{array}{c}\begin{array}{c}\text { Número } \\
\text { total semillas }\end{array} \\
\end{array}$ & $\begin{array}{c}\text { Precio/1000 } \\
\text { semillas (USD) }\end{array}$ & $\begin{array}{c}\text { Beneficio Bruto } \\
\left(1000 \mathrm{~m}^{2}\right)\end{array}$ & Costo $\left(1000 \mathrm{~m}^{2}\right)$ & BN (USD) & $\begin{array}{c}\begin{array}{c}\text { Relación } \\
\text { B/C }\end{array} \\
\end{array}$ & Rentabilidad \\
\hline Con poda & 2640 & 303.00 & 799920.00 & 17.5 & 13998.60 & 1000.00 & 12998.60 & 13.00 & Rentable \\
\hline Sin poda & 2640 & 85.00 & 224400.00 & 17.5 & 3927.00 & 1000.00 & 2927.00 & 2.93 & Rentable \\
\hline
\end{tabular}

Los principales métodos para crear nuevos cultivares en especies autógamas son: la introducción, selección e hibridación ${ }^{\underline{31}}$. Los métodos de selección aplicados son la selección en masa o masal y la selección de líneas puras ${ }^{31, \underline{32}}$. Una línea pura no es más que una progenie descendiente únicamente por autofecundación de una planta individual homocigótica, los representantes de una línea pura son todos idénticos en cuanto a sus características ${ }^{31}$. La selección de líneas puras se practica en poblaciones segregantes después de la hibridación artificial de dos cultivares. El método de hibridación consiste en cruzar dos cultivares o líneas puras y seleccionar en la descendencia los segregantes que contengan la combinación de las mejores características de los progenitores $\underline{31, \underline{32}}$.

Hoy en día la mejora genética del melón se enfoca en la obtención de híbridos de gran vigor, debido a la heterosis que se observa para la mayor parte de los caracteres de interés agronómico ${ }^{33,34}$. Las técnicas de hibridación se explotan ampliamente y permiten introducir distintos genes de interés económico en las plantas. Hoy en día, no existe ninguna teoría concluyente sobre cómo ocurren las interacciones génicas no aditivas aprovechadas en la hibridación comercial, las cuales conducen a una sobre manifestación de los híbridos con respecto a los padres en cuanto a crecimiento, vitalidad, fertilidad, 
adaptabilidad al medio y otros caracteres y cualidades, las cuales han sido designadas con el nombre

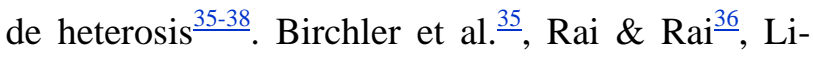
ppman \& Zamir $^{37}$ Springer \& Stupar ${ }^{38}$. Marquéz ${ }^{32}$, plantean que la hibridación en las plantas es el método genotécnico que aprovecha la generación $F_{1}$ proveniente del cruzamiento entre dos poblaciones parentales (hembras y machos), las cuales pueden ser líneas endogámicas, cultivares de polinización libre, o cultivares sintéticos.

Se plantea que para iniciar un plan de obtención de híbridos se requieren, ante todo, tener conocimientos acerca de la biología de la especie con que se trabaja y el modo de herencia genética de aquellos caracteres con los cuales se desea dotar a los nuevos genotipos $^{34,39}$.

En nuestra investigación, dispusimos de dos líneas parentales puras (con alto grado de homocigosis), de la Empresa Harris Moran, usadas en la producción de híbridos $F_{1}$ de melón, que son comercializadas en diversas partes del mundo. $\mathrm{Al}$ respecto, se menciona que se requiere al menos seis ciclos de autofecundaciones sucesivas de los parentales para llegar de 98 a $99 \%$ de homocigosis ${ }^{40}$.

Sin embargo, no conocíamos el comportamiento de estos parentales bajo las condiciones de Puerto La Boca. Pudimos observar que los frutos obtenidos de la cruza entre los dos parentales, mejoraron sustancialmente con la poda del progenitor femenino, esta práctica nos permitió obtener semillas híbridas $F_{1}$ de melón de buena calidad. Esta innovación en la producción de semilla de calidad de melón, es una alternativa factible de negocio para los productores, que podrían producir semilla de calidad para la empresa Harry Morán. Así, Puerto La Boca podría convertirse en un sitio potencial, para proveer semilla híbrida de calidad en todas las épocas del año, debido a que países productores como China y otros, no pueden producir en todas las épocas por las condiciones de clima. Asimismo, esta actividad, permitiría innovar la matriz productiva, al usar de manera más eficiente y efectiva los invernaderos disponibles, que garantizaría un negocio sostenible para los productores.

La importancia de la creación de híbridos con resistencia a enfermedades y elevada productividad es evidente y constituye un objetivo primordial de cualquier programa de mejora genética que se pretenda llevar a cabo en los próximos años ${ }^{30,41}$. Esto fue evidenciado en nuestra investigación, obtuvimos semillas híbridas $F_{1}$ de melón de alta calidad genética. Sin embargo, será importante lograr un convenio comercial con la empresa Harry Moran, para acceder a sus líneas parentales, con la finalidad de producir semilla híbrida y garantizar su venta a la empresa. Cabe mencionar, que la empresa desarrolló durante muchos años sus propias líneas parentales, que podrían estar disponibles, siempre y cuando se respete la propiedad intelectual. Bravo ${ }^{41}$ menciona, que los derechos de propiedad intelectual constituyen al momento el eslabón más importante para asegurar el control sobre las semillas por parte de las empresas transnacionales.

La producción de semillas híbridas en el Ecuador representa una industria nueva, en la cual existe poca competencia, puesto que al momento solo dos instituciones se dedican a la producción de híbridos, tales como Agripac y el INIAP ${ }^{42}$. A nivel local las semillas de mayor demanda son las de maíz, básicamente su diferencia con la semilla tradicional radica en la resistencia que posee frente a plagas, virus y condiciones climáticas que permiten un mayor rendimiento productivo, las empresas obtienen más producción a un mismo costo, siendo capaces de mejorar sus márgenes de rentabilidad. 
Según Megson-Villao ${ }^{43}$ las empresas multinacionales normalmente hacen el negocio de semilla híbrida en países en desarrollo, porque les representa un menor costo en mano de obra, y aprovechan las condiciones favorables del clima, como es el caso de Ecuador, donde es propicio el crecimiento de melón ${ }^{44}$. Con la producción de semilla híbrida en Ecuador se estaría reemplazando el mercado de China y Nigeria, que son los mayores productores de semilla actualmente ${ }^{45}$.

Observamos que la práctica de podar el parental femenino, una vez hecho los cruzamientos, mejora el volumen de fruto, el peso de fruto, el número de semilla y el peso de semilla. Además, observamos un buen comportamiento heterótico del hibrido. Esto concuerda con los resultados reportados por Yilmaz \& Sari ${ }^{46}$, quienes observaron que la mayor heterobeltiosis $(184.23 \%)$ y las tasas de heterosis (184.55\%) se produjeron en forma temprana en combinaciones hibridas de genotipos. Asimismo, mencionaron una superioridad del híbrido individual en desarrollo de planta, rendimiento total y precocidad.

\section{Fuente de financiamiento}

Grant PROG-003-PROY-001-DIP-2017 a JGO de la Universidad Estatal del Sur de Manabí.

\section{Conflictos de intereses}

Los autores declaran que esta investigación fue realizada en la Universidad Estatal del Sur de Manabí (Cantón Jipijapa) y no presenta conflictos de interés.

\section{Agradecimientos}

47
Los autores agradecen el apoyo financiero y las facilidades proporcionadas por la Universidad Estatal del Sur de Manabí (UNESUM). También agradecemos a los agricultores de Puerto La Boca, a todos los estudiantes involucrados en esta investigación y a la Empresa Harris Morán de USA, por proveer los materiales de estudio.

\section{Consideraciones éticas}

La aprobación de la investigación por la Dirección de Investigación y Posgrado, el Comité de Ética, y el Comité de Investigación de la Carrera de Agropecuaria de la Universidad Estatal del Sur de Manabí (UNESUM), (Cantón Jipijapa), se siguió las pautas establecidas por estas instancias.

\section{Aporte de los autores en el artículo}

Julio Gabriel-Ortega, Planeación del experimento, análisis estadístico y sistematización e interpretación de la información. Gema Burgos-López, Toma de datos y sistematización e interpretación de la información. Nora Barahona-Cajape, Toma de datos y sistematización e interpretación de la información. Carlos Castro Piguave, Planeación del experimento, revisión del artículo. Máximo Vera Tumbaco, Planeación del experimento, revisión del artículo. Morán Morán Jessica, Planeación del experimento, revisión del artículo.

\section{Literatura citada}

1. International Plant Genetic Resources Institute. Descriptors for Melon (Cucumis melo L.) [Internet]. Rome: International Plant Genetic Resources Institute; 2003 [citado 19 de enero de 2021]. 77 p. Recuperado a partir de https://www.bioversityinternational.org/fileadmin 
L_migrated/uploads/tx

news/Descriptors_for_melon_Cucumis_melo_ L. $906 . p d f$

2. Pitrat M, Melon. In: Prohens J, Nuez F, editors. Vegetals I. Handbook of plant breeding. New York: Springer; 2008. p. 238-315. DOI: https:// doi.org/10.1007/978-0-387-30443-4_9

3. Thakur H, Sharma S, Thakur M. Recent trends in muskmelon (Cucumis melo L.) research: an overview. J Hortic Sci Biotechnol 2019;94(4):533-47. https://doi.org/10.1080/14620316.2018.1561214

4. Kroon GH, Custers JBM, Kho YO, den Nijs APM, Varekamp HQ. Interspecific hybridization in Cucumis (L.). I Need for genetic variation, biosys-tematic relations and possibilities to overcome crossability barriers. Euphytica 1979;28:723-8.

DOI: https://doi.org/10.1007/BF00038940

5. Kirkbride Jr JH. Biosystematic monograph of the genus Cucumis (Cucurbitaceae). botanical identification of cucumbers and melons [Internet]. North Carolina: Parkway Publishers; 1993 [citado 22 de octubre de 2019]. 34 p. Recuperado a partir

de: file:///C:/Users/usuario/Downloads/kirkbridge 1993 OCR.pdf

6. Tzitzikas EN, Monforte AJ, Fatihi A, Kypriotakis Z, Lacovides TA, Loannides IM, et al. Genetic diversity and population structure of traditional greek and cypriot melon cultigens (Cucumis melo L.) based on simple sequence repeat variability. HortScience 2009;44(7):1820-4. DOI: https://doi. org/10.21273/HORTSCI.44.7.1820

7. Casi 30.000 millones de kilos de melón se producen en el mundo, la mitad en China [Internet]. Hortoinfo-Diario Digital de Actualidad Hortofrutícola. 2021 [citado 01 de enero 2021]. Recupe- rado a partir de http://www.hortoinfo.es/index. php/5338-prod-mund-melon-240217

8. Beaulieu JC, Lea JM, Eggleston G, Peralta Inga Z. Sugar and organic acid variations in commercial cantaloupes and their inbred parents. J Amer Soc Hort Sci 2003;128(4):5321-6. DOI: https://doi. org/10.21273/JASHS.128.4.0531

9. Ramírez Rosales GK. Análisis económico de la producción de sandía (Citrullus lanatus) injertada sobre patrones de calabaza en la provincia de Santa Elena [tesis licenciatura]. [La libertad]: Universidad Estatal Península de Santa Elena; 2014 [citado 26 de octubre de 2019]. Recuperado a partir de: https://repositorio.upse.edu.ec/xmlui/ handle/46000/2250

10.Borbor Quirumbay EJ, Domínguez Rodríguez GE. Empleo de tecnologías limpias para el manejo de problemas fitosanitarios en el cultivo de melón Cucumis melo L. comuna Río Verde, Santa Elena [tesis licenciatura]. [La libertad]: Universidad Estatal Península de Santa Elena; 2010 [citado 26 de octubre de 2019]. Recuperado a partir de: https://repositorio.upse.edu.ec/xmlui/handle/4600 $\underline{0 / 910}$

11.Carrillo Alvarado R, Carvajal Mera T, Valarezo Cely O, Cañarte Bermúdez E, Mendoza García A, Mendoza Zambrano $\mathrm{H}$, et al. Manual de buenas practicas agrícolas y estimación de costos de producción para cultivos de ciclo corto en Manabí [Internet]. Portoviejo: Instituto Nacional Autonomo de Investigaciones Agropecuarias; 2010 [citado 26 de octubre de 2019]. Manual No. 84. Recuperado a partir de: https://repositorio.iniap.gob. ec/handle/41000/1294 
12.Robinson RW, Decker Walters D S. Cucurbits crop production science in horticulture series, Wallingford, U.K.: CAB International; 1997. p. 65-6, 152-62.

13.Pitrat M, Chauvet M, Foury C. Diversity, history and production of cultivated cucurbits. Acta Hortic 1999;492:21-8. DOI: https://doi.org/10.17660/ ActaHortic.1999.492.1

14.Deleu W, Esteras C, Roig C, González To M, Fernández Silva I, Gonzalez Ibeas D, et al. A set of EST-SNPs for map saturation and cultivar identification in melon. BMC Plant Biol 2009;9:90. DOI: https://doi.org/10.1186/14712229-9-90

15.Esteras C, Formisano G, Roig C, Diaz A, Blanca $\mathrm{J}$, Garcia Mas J, et al. SNP genotyping in melons: genetic variation, population structure, and linkage disequilibrium. Theor Appl Genet 2013; 126(5):1285-303. DOI: https://doi.org/10.1007/ $\underline{\text { s00122-013-2053-5 }}$

16.Barman A, Mitra R, Singh DK, Anshumali CE. Diversity among Indigenous germplasms of melon (Cucumis melo L.) through seed protein profiling pattern. Vegetos 2015;28(3):1-7. DOI: https://doi.org/10.5958/2229-4473.2015.00060.9

17.Luan F, Sheng Y, Wang Y, Staub JE. Performance of melon hybrids derived from parents of diverse geographic origins. Euphytica 2010;173(1):1-16.

DOI: https://doi.org/10.1007/s10681-009-0110-6

18. Singh SP, Sharma JR. Genetic improvement of pyrethrum: 4. selective divergence, heterosis and potential hybrid clones. Theor Appl Genet 1989; 78(6):841-6. DOI: https://doi.org/10.1007/BF00 $\underline{266668}$

19. Teklewold A, Becker HC. Comparison of phenotypic and molecular distances to predict heterosis and F1 performance in Ethiopean mustard (Brassica carinata A. Braun). Theor Appl Genet 2006; 112(4):752-9. DOI: https://doi.org/10.1007/s0012 2-005-0180-3

20.Xiao J, Li J, Yuan L, McMocouch SR, Tankley SD. Genetic diversity and its relationship to hybrid performance and heterosis in rice as revealed by PCR-based markers. Theor Appl Genet 1996; 92(6):637-43. DOI: https://doi.org/10.1007/BF00 $\underline{226083}$

21.Ferreira MAJ, Braz LT, Queiroz MA, Churata Masca MGC, Vencovsky R. Capacidade de combinacao em sete populacoes de melancia. Pesq Agropec Bras 2002;37(7):963-70. DOI: https:// doi.org/10.1590/S0100-204X2002000700010

22.Souza FFD, Queiroz MAD, Dias RDCDS. Capacidade de combinacao entre linhas tetraploides e diploides do melancia. Hortic Bras 2002; 20(4):654-8. DOI: https://doi.org/10.1590/S0102$\underline{05362002000400029}$

23.Gabriel Ortega J, Pereira Murillo E, Ayón Villao F, Castro Piguave C, Delvalle García I, Castillo JA. Development of an ecological strategy for the control of downy mildew (Pseudoperonospora cubensis) in cucumber cultivation (Cucumis sativus L.). Bionatura 2020;5(2):1101-5. https://doi. org/10.21931/RB/2020.05.02.3

24. Secretaria Nacional de Planificación y Desarrollo. Plan de Ordamiento y Desarrollo Territorial de Puerto Cayo 2015 [Internet]. Puerto Cayo: Secretaria Nacional de Planificación y Desarrollo; 2015 [citado 22de octubre de 2019]. 231 p. Recuperado a partir de: http://app.sni.gob.ec/snilink/sni/PORTAL_SNI/data_sigad_plus/sigadplu sdocumentofinal/1360043010001_PDOT\%20PUERTO\%20 CAYO\%20FINAL_31-10-2015_00-04-31.pdf 
25.Gabriel J, Castro C, Valverde A, Indacochea B, Diseños experimentales: Teoría y práctica para experimentos agropecuarios. Grupo COMPAS, Universidad Estatal del Sur de Manabí (UNESUM), Jipijapa, Ecuador. 2017. 116 p. Recuperado a partir de http://142.93.18.15:8080/jspui/handle/123 456789/116

26.SAS University [Internet]. An Introduction to SAS University Edition; 2019 [citado 26 de octubre de 2019]. Recuperado a partir de: https:// www.oreilly.com/library/view/an-introductionto/9781629600079/

27. Moreno JM. Fórmulas y volúmenes para la determinación de áreas y volúmenes. Guiniguada 2000; 9(8/9):291-3187.

28.Boardman AE, Weimer DL Vining AR Greenberg DH. Cost-benefit Analysis: Concepts and Practice. $3^{\text {rd }}$ ed. Columbia: Cambridge; 2006. 586 p.

29.Berlanga Silvente V, Rubio Hurtado MJ. Clasificación de pruebas no paramétricas. Cómo aplicarlas en SPSS. REIRE 2012;5(2):101-13. DOI: https://doi.org/0.1344/reire2012.5.2528

30.Lemus Islas Y, Hernández Salgado JC. Situación actual del mejoramiento genético del melón para la resistencia al Mildiu pulverulento de las cucurbitáceas. Tema de Cienc y Tecnol 2003;7(9):2536.

31.Allard RW. Principles of Plant Breeding. $2^{\text {nd }}$ ed. New York: John Wiley \& Sons; 1999. 498 p

32.Márquez F. Introducción a la hibridación en genotécnia vegetal (Tomo I). México: A.G.T Editor, S.A.; 19988. p. 1-16.

33.Fernández Silva I, Moreno E, Eduardo I, Arús P, Álvarez JM, Monforte AJ. On the genetic control of heterosis for fruit shape in melon (Cucumis
Melo L.). J Hered 2009;100(2):229-35. DOI: https://doi.org/10.1093/jhered/esn075

34.Reyna Trujillo TJ, Vega León M, Gordillo Ortuño M. Producción, postproducción y agrotecnias de semillas, hortalizas y frutas. Coadyuvantes en la seguridad alimentaria en México y Cuba [Internet]. Coyoacán: Universidad Nacional Autónoma de México; 2016 [citado 22-de octubre de 2019]. 265 p. Recuperado a partir de: http://www.publicaciones.igg.unam.mx/index.ph p/ig/catalog/view/ 85/85/258-1

35.Birchler JA, Auger DL, Riddle NC. In search of a molecular basis of heterosis. Plant Cell 2003; 15(10):2236-9. DOI: https://doi.org/10.1105/tpc. $\underline{151030}$

36.Rai N, Rai M. Heterosis breeding in vegetable crops. New Delhi: New India Publishing Agency, 2006. 546 p.

37.Lippman ZB, Zamir D. Heterosis: revisiting the magic. Trends Genet 2007;23(2):60-6. DOI: https://doi.org/10.1016/j.tig.2006.12.006

38. Springer NM, Stupar RM. Allelic variation and heterosis in maize: how do two halves make more than a whole? Genome Res 2007;17(3):264-75.

DOI: https://doi.org/10.1101/gr.5347007

39.Gómez-Guillamón ML. Mejora genética del melón (Cucumis melo L.). Estación Experimental "La Mayora": Consejo Superior de Investigaciones Científicas; 1985.

40.González MC. Curso internacional de verano: Biología y mutagénesis. La Habana: Instituto Nacional de Ciencias Agrícolas; 1995. s/p.

41.Bravo E. Normativas sobre semillas en América Latina al servicio del control corporativo [Internet]. Quito: Red por una América Latina Libre de Transgénicos; 2015 [citado 22-de octubre de 
2019]. 56 p. Recuperado a partir de: https:// www.rallt.org/PUBLICACIONES/Normativas\% 20sobre\%20semillas.pdf

42. Monge Pérez JE. Evaluación de 70 genotipos de melón (cucumis melo L.) cultivados bajo invernadero en Costa Rica. InterSedes 2016;17(36):241.

DOI: https://doi.org/10.15517/ISUCR.V17136.26944

43.Megson Villao RM. Proyecto para la producción y exportación de semillas híbridas de pepino [tesis licenciatura]. [Samborondón]: Universidad Espíritu Santo; 2013 [citado 26 de octubre de 2019]. Recuperado a partir de: http://201.159.223.2/ bitstream/123456789/1177/1/TESIS\%20ROBER TA\%20MEGSON.pdf

44.Dried melon seed [Internet]. Global SourcesReliable exporters: find them and meet them. 2019 [citado 5 de marzo de 2019]. Recuperado a partir de: https://chinasuppliers.spanish.globalsources.com/ china-suppliers/Dried-Melon-Seed.htm

45.Melon Seed [Internet]. Tridge. 2019 [citado 5 de marzo de 2019]. Recuperado a partir de: https:// www.tridge.com/intelligences/melon-seed
46. Yilmaz N, Sari N. Heterosis effect on plant growth fruit, yield and quality in single, triple and double crosses of melon (Cucumis melo var. cantalu-pensis) Hybrids. En: Yilmaz N, Sari N, editors. Proceeding of the Xth EUCARPIA Meeting on Genetics and Breeding of Cucurbitaceae. 2012 [Internet]. Antalya, Turkey; 2012. p. 535543. Recuperado a partir de: https://www.researchgate. net/publication/260638876_Heterosis_Effect_On Plant Growth Fruit Yield And Quality In _Single_Triple_And_Double_Crosses_Of_Melon Cucumis Melo Var Cantalupensis Hybrids

\section{Nota del Editor:}

Journal of the Selva Andina Research Society (JSARS) se mantiene neutral con respecto a los reclamos jurisdiccionales publicados en mapas y afiliaciones institucionales. 